G. Ikegami

Nagoya Math. J.

Vol. 67 (1977), 15-34

\title{
EXISTENCE OF REGULAR COVERINGS ASSOCIATED WITH LEAVES OF CODIMENSION ONE FOLIATIONS
}

\author{
GIKŌ IKEGAMI
}

\section{§1. Statement of results}

In this paper we are concerned with transversely orientable codimension one foliations. Let $\mathscr{F}$ be a $C^{r}$-foliation as above in a smooth manifold $M, r \geqq 1$, and let $F_{0}$ be a closed leaf of $\mathscr{F}$. A neighborhood $U$ of $F_{0}$ is called a bicollar of $F_{0}$ in this paper if there is a normal line bundle $\nu: U \rightarrow F_{0}$ with respect to a fixed Riemannian metric on $M$ such that each fibre of $\nu$ is transverse to $\mathscr{F}$. For a bicollar $U$ of $F_{0}, U_{+}$ $=F_{0} \cup\left(\right.$ a component of $\left.U-F_{0}\right)$ is called a collar of $F_{0}$. A leaf $F \in \mathscr{F}$ is said to be asymptotic to $F_{0}$ in $U_{+}$if $F \cap V \neq \phi$ for any neighborhood $V$ of $F_{0}$ in $U_{+}$. Let $F_{V}$ be a leaf asymptotic to $F_{0}$ of the restricted foliation $\mathscr{F} \mid V$, where $V$ is a neighborhood of $F_{0}$ in $U_{+}$. A plaque of $F$ is a leaf of $F \mid N$ diffeomorphic to an open $(n-1)$-ball, where $N$ is a sufficiently small open $n$-ball in the $n$-manifold $M$. A $C^{r}$-covering $\tilde{\nu}: \tilde{F}$ $\rightarrow F_{0}$ is said to be associated with $F_{V}$ if there is an injection $i: F_{V} \rightarrow \tilde{F}$ such that $i=\nu \mid F_{V}$ and that $i$ maps any plaque of $F_{V} C^{r}$-diffeomorphically into $\tilde{F}$. The one sided holonomy group $\Phi_{+}\left(F_{0}\right)$ of $F_{0}$ is the holonomy group of $F_{0}$ defined by the restricted foliation $\mathscr{F} \mid U_{+}$.

The main purpose of this paper is to prove Theorem 2, which is an existence theorem of associated regular coverings. Theorem 1 is used in the proofs of Theorem 2 and Theorem 5. Theorem 3 and Theorem 4 are the properties of associated regular coverings. As an application we show Theorem 5, which is an unstability theorem of foliations.

THEOREM 2. Let $\mathscr{F}$ be a transversely orientable $C^{r}$-foliation of codimension one, $r \geqq 1, F_{0}$ be an orientable closed leaf of $\mathscr{F}$, and let $U_{+}$ be a collar of $F_{0}$. Suppose that the one sided holonomy group $\Phi_{+}\left(F_{0}\right)$ is abelian. Then there is a neighborhood $V_{0}$ of $F_{0}$ in $U_{+}$such that any

Received February 10, 1975.

Revised November 5, 1976. 
neighborhood $V$ of $F_{0}$ in $V_{0}$ satisfies the followings.

For each asymptotic leaf $F$ to $F_{0}$ in $U_{+}$let $F_{V}$ be an asymptotic leaf of $\mathscr{F} \mid V$ to $F_{0}$ contained in $F$. Then, an unique (in the sense of the equivalence of coverings) $C^{r}$-regular covering $\tilde{\nu}: \tilde{F} \rightarrow F_{0}$ is associated with $F_{V}$ and $\nu_{*}\left(\pi_{1}\left(F_{V}\right)\right)=\tilde{\nu}_{*}\left(\pi_{1}(\tilde{F})\right)$ in $\pi_{1}\left(F_{0}\right)$. Furthermore, the equivalence class of $\tilde{\nu}$ does not depend on $V$, and so an unique normal subgroup $G(F)=\nu_{*}\left(\pi_{1}\left(F_{V}\right)\right)$ of $\pi_{1}\left(F_{0}\right)$ is associated with $F$.

$\tilde{\nu}$ and $G(F)$ are considered as invariants on the behavior of $F$ in a neighborhood of $F_{0}$ in $U_{+}$. There is an example of $\mathscr{F}, F_{0}$, and an asymptotic leaf $F$ to $F_{0}$ such that, for any one sided neighborhood $V$ of $F_{0}$, no regular covering is associated with $F_{V}$.

THEOREM 1. Suppose that $\mathscr{F}, F_{0}$, and $U_{+}$satisfy the same conditions as Theorem 2. Then, there are connected orientable codimension one submanifolds $N_{1}, \cdots, N_{\ell}$ of $F_{0}$ satisfying the followings.

(i) $F_{0}-N_{1} \cup \cdots \cup N_{\ell}$ is connected.

(ii) Let $F_{*}$ be the manifold obtained by cutting open $F_{0}$ along $N_{1}, \cdots, N_{\ell}$, and let $g: F_{*} \rightarrow F_{0}$ be the map pasting $F_{*}$ on $F_{0}$ naturally. (There are definitions of $F_{*}$ and $g$ in §3.) Thus $\partial F_{*}=\cup_{i=1}^{\epsilon} N_{i}^{\prime} \cup N_{i}^{\prime \prime}$, $g^{-1}\left(N_{i}\right)=N_{i}^{\prime} \cup N_{i}^{\prime \prime}$, and $g\left(N_{i}^{\prime}\right)=N_{i}=g\left(N_{i}^{\prime \prime}\right)$. Then, there are injective diffeomorphisms $f_{i}:[0, \varepsilon] \rightarrow[0, \varepsilon], i=1, \cdots, \ell$ with the following properties.

(a) $f_{i}(0)=0$ and $f_{i} f_{j}(t)=f_{j} f_{i}(t)$ for any $i, j=1, \cdots$, $l$ and $t$ such that $f_{i} f_{j}(t)$ and $f_{j} f_{i}(t)$ are defined. (b) Denote by $X_{f}$ the quotient manifold obtained from $F_{*} \times[0, \varepsilon]$ by identifying $(x, t) \in N_{i}^{\prime} \times[0, \varepsilon]$ and $\left(x, f_{i}(t)\right)$ $\in N_{i}^{\prime \prime} \times[0, \varepsilon]$ for all $i=1, \cdots, l$ and $t \in[0, \varepsilon]$. By the commutativity of $f_{i}$ and $f_{j}, X_{f}$ is well defined. The product foliation of $F_{*} \times[0, \varepsilon]$ induces a foliation $\mathscr{F}_{f}$ on $X_{f}$. Then, there is a neighborhood $V$ of $F_{0}$ in $U_{+}$ such that there is a leaf preserving $C^{r}$-diffeomorphism from $V$ onto $X_{f}$. (c) The germs of $f_{1}, \cdots, f_{\ell}$ at 0 generate $\Phi_{+}\left(F_{0}\right)$. Moreover, if $\operatorname{dim} F_{0}$ $>2$, they are chosen so that the germs of $f_{1}, \cdots, f_{\ell}$ are a basis of $\Phi_{+}\left(F_{0}\right)$.

The following results are consequence of Theorem 1 and Theorem 2.

THEOREM 3. Let $\mathscr{F}$ be a transversely orientable $C^{1}$-foliation of codimension one, and let $F_{0}$ be an orientable closed leaf of $\mathscr{F}$. Suppose that $\pi_{1}\left(F_{0}\right)=Z^{m} \times G$ for a finite group $G$ and that $\left\{\log h_{\alpha_{1}}^{\prime}, \cdots, \log h_{\alpha_{m}}^{\prime}\right\}$ 
is rationally independent for a basis $\alpha_{1}, \cdots, \alpha_{m}$ of $Z^{m}$, where $h_{\alpha_{i}}^{\prime}$ is the derivative of the holonomy of $\alpha_{i}$.

Then there are collars $U_{+}$and $U_{-}$in the both sides of $F_{0}$ such that any leaf meeting $U_{\sigma}$ is asymptotic to $F_{0}$ in $U_{\sigma}$ and that, for any neighborhood $V$ of $F_{0}$ in $U_{\sigma}$ and for any $F \in \mathscr{F}$ meeting $U_{\sigma}$, an unique regular covering $\tilde{F}$ with $\pi_{1}(\tilde{F}) \cong G$ is associated with $F_{V}$. Here $\sigma$ denotes + or 一.

THEOREM 4. Let $\mathscr{F}$ be a transversely orientable codimension one foliation of class $C^{r}$, for $r \geqq 2$, and let $F_{0}$ be an orientable closed leaf of $\mathscr{F}$. Suppose that the holonomy group $\Phi\left(F_{0}\right)$ of $F_{0}$ is abelian and that there is $\tilde{f} \in \Phi\left(F_{0}\right)$ such that the derivative $\tilde{f}^{\prime}$ of $\tilde{f}$ at 0 satisfies $\tilde{f}^{\prime} \neq 1$.

Then, there is a bicollar $U=U_{+} \cup U_{-}$of $F_{0}$ satisfying the followings. Let $\sigma$ denote + or -. (i) Any leaf meeting $U_{\sigma}$ is asymptotic to. $F_{0}$ in $U_{\sigma}$. (ii) For any neighborhood $V$ of $F_{0}$ in $U_{\sigma}$ and for any leaf $F$ meeting $U_{o}$, an unique regular covering $\tilde{\nu}$ of $F_{0}$ is associated with $F_{V}$ and the normal subgroup $G(F)$ of $\pi_{1}\left(F_{0}\right)$ is well defined. Moreover, (iii). г and $G(F)$ do not depend on $U_{+}, U_{-}$, and $F$.

This theorem shows that, under the above assumptions, all leaves near $F_{0}$ in a collar are in the same situation and $\mathscr{F}\left|U_{+}, \mathscr{F}\right| U_{-}$have the same structure.

Let $F$ be a closed submanifold of $M$, and let $\mathscr{F}, \mathscr{F}^{\prime}$ be foliations on a neighborhood of $F$ in $M$ having $F$ as a leaf. We say that $\mathscr{F}$ and $\mathscr{F}^{\prime}$ are locally equivalent at $F$, if there are neighborhoods $U$ and $U^{\prime}$ of $F$ such that there is a homomorphism from $U$ onto $U^{\prime}$ mapping any leaf of $\mathscr{F} \mid U$ onto a leaf of $\mathscr{F}^{\prime} \mid U^{\prime}$.

Let $\mathscr{F}_{F}^{1}$ be the set of germs at $F$ of codimension $k C^{1}$-foliations $\mathscr{F}$ defined on neighborhoods $U_{\mathscr{F}}$ of $F$ in $M$ such that $\mathscr{F}$ has $F$ as a leaf, and let $\mathscr{F}_{F}^{1}$ have a suitable topology defined by the germ of the section into the Grassmannian which defines the foliation. H. Levine and M. Shub show an unstability theorem [2] as follows: If $\pi_{1}(F)$ has the form $Z^{m} \times G$ for $m>1$ and an arbitrary group $G$, there are no stable elements in $\mathscr{F}_{F}^{1}$ with respect to local equivalence at $F$.

Here, we show an unstability theorem for foliations defined on a fixed neighborhood $U$ of $F$ in $M$. Let $\operatorname{Fol}_{F}^{r}(U)$ be the space of $C^{r}$ foliations $\mathscr{F}$ of codimension one defined on a neighborhood $U$ of $F$ in $M$ such that $\mathscr{F}$ has $F$ as a leaf. $\operatorname{Let}^{\operatorname{Fol}_{F}^{r}}(U)$ have the $C^{r}$-topology defined 
in [1] using the charts $\left\{\varphi: I^{n-1} \times I \rightarrow M^{n}\right\}$.

THEOREM 5. Let $F$ be an orientable closed submanifold of $M$ of codimension one such that $\pi_{1}(F)=Z^{m} \times G$ for $m>1$ and a finite group $G$. Let $\mathscr{F}$ be a transversely orientable codimension one foliation of class $C^{r}$ on a neighborhood of $F$ in $M$ with $F$ as a leaf. Then,

(i) if $r=2$, there is a neighborhood $U$ of $F$ such that for any neighborhood $N$ of $\mathscr{F} \mid U$ in $\operatorname{Fol}_{F}^{1}(U)$ there is $\mathscr{F}^{\prime}$ in $N$ which is not locally equivalent at $F$ to $\mathscr{F}$. Moreover,

(ii) if $r>2$, assume that there is $\alpha$ in $\pi_{1}(F)$ such that $\left|h_{\alpha}^{\prime}\right| \neq 1$, where $h_{\alpha}^{\prime}$ is the derivative of the holonomy of $\alpha$. Then, the same result as (i) holds for $\mathrm{Fol}_{F}^{r-1}(U)$.

In the preparation for this research the papers, [4] of Nishimori and [3] of Nakatsuka, were very helpful to the author.

\section{§. Preparation for Theorem 1}

This section will be in the version of class $C^{\infty}$. Let $M$ be an oriented $n$-manifold, $n \geqq 3$, and let $N$ be an oriented closed smooth submanifold of $M$ with codimension one. Let $F: B^{n-1} \times I \rightarrow M$ be an orientation preserving embedding such that $F\left(B^{n-1} \times I\right) \cap N=F\left(B^{n-1} \times \partial I\right)$, where $B^{n-1}$ denotes an $(n-1)$-ball in $R^{n-1}$ with origin $0, I=[0,1]$, and $\partial$ denotes the boundary. We obtain an $(n-1)$-submanifold

$$
N_{*}=\left\{N-\operatorname{int} F\left(B^{n-1} \times \partial I\right)\right\} \cup F\left(\partial B^{n-1} \times I\right) .
$$

By smoothing the corners, $N_{*}$ can be regarded as a smooth manifold. Define a simple arc $f: I \rightarrow M$ by $f(t)=F(0, t), t \in I$. We shall say that $N_{*}$ is obtained from $N$ by attaching a 1-handle along a simple arc $f$. If the intersection number of $N$ and $f$ is zero, $N_{*}$ is orientable. In this case we assume that $N_{*}$ has the orientation compatible with that of $N$. Then, $\left[N_{*}\right]=[N]$ in $H_{n-1}(M ; Z)$, where [ ] denotes the homology class.

LEMMA 1. Let $M$ be an oriented manifold of dimension $n \geqq 3$, and let $N^{\prime}$ be a connected oriented closed $(n-1)$ submanifold of $M$. Then, for a simple closed path $c$ in $M$ which intersects $N^{\prime}$ at finite points, there is a connected oriented closed $(n-1)$ submanifold $N$ of $M$ satisfying the following conditions.

(i) $[N]=\left[N^{\prime}\right]$ in $H_{1}(M ; Z)$. 
(ii) $N$ intersects $c$ at only $|[c] \cdot[N]|$ points.

(iii) For a small neighborhood $U$ of $c$ in $M, N$ is included in $N^{\prime} \cup U$.

Proof. We may assume $[c] \cdot\left[N^{\prime}\right] \geqq 0$ and that $N^{\prime}$ intersects with $c$ transversely at more than $[c] \cdot\left[N^{\prime}\right]$ points, $x_{1}=c\left(t_{1}\right), \cdots, x_{r}=c\left(t_{r}\right), 0<t_{1}$ $<\ldots<t_{r}<1$. We construct by induction on $r$ the desired manifold $N$. There is $i$ such that $1 \leqq i \leqq r-1$ and that the intersection number of $N^{\prime}$ and $c \mid\left[t_{i}, t_{i+1}\right]$ is zero. By attaching a 1-handle to $N^{\prime}$ along the simple subarc $c \mid\left[t_{i}, t_{i+1}\right]$, we obtain $N_{*}^{\prime}$ which intersects at $(r-2)$ points and with $\left[N_{*}^{\prime}\right]=\left[N^{\prime}\right]$. Then $N_{*}^{\prime}$ has the inductive property.

LEMMA 2. Let $N \subset M$ be a pair of oriented connected manifolds of codimension one. If there is $\gamma$ in $H_{1}(M ; Z)$ such that the intersection number $\gamma \cdot[N]$ is 1 , then $M-N$ is connected.

Proof. First, we show that there is a closed path $u: I \rightarrow M, u(0)$ $=u(1)$, such that $u$ intersects with $N$ at a single point. Let $c$ be any closed path with $[c]=\gamma$. We may assume that $c$ meets $N$ transversely, and hence $c$ meets $N$ at finitely many points, $x_{1}=c\left(t_{1}\right), \cdots, x_{r}=c\left(t_{r}\right)$, $0<t_{1}<\ldots<t_{r}<1$. We shall construct by induction on $r$ a closed path $u$ as above. We may assume $r \geqq 3$. There is $i$ with $1 \leqq i \leqq r-1$ such that the intersection number of $N$ and $c \mid\left[t_{i}, t_{i+1}\right]$ is zero. Since $N$ is connected there is a path $d$ from $x_{i}$ to $x_{i+1}$ in $N$. Let $\varepsilon$ be a sufficiently small positive real number. Then, we can take a path $d^{\prime}$ from $c\left(t_{i}-\varepsilon\right)$ to $c\left(t_{i+1}+\varepsilon\right)$ along $d$ so that $d^{\prime}$ does not intersect with $N$. $c\left(\left[0, t_{i}-\varepsilon\right]\right) \cup\left(\right.$ image $\left.d^{\prime}\right) \cup c\left(\left[t_{i+1}+\varepsilon, 1\right]\right)$ is an image of a path $c^{\prime}: I \rightarrow M$ which meets $N$ at $(r-2)$ points. Moreover, we have $\left[c^{\prime}\right] \cdot[N]=\gamma \cdot[N]$ $=1$, where $\left[c^{\prime}\right]$ denotes the homology class of $c^{\prime}$. Then $c^{\prime}$ has the inductive property, and therefore $u$ is constructed.

For any two points $p_{0}$ and $p_{1}$ in $M-N$ there is a path $c$ from $p_{0}$ to $p_{1}$. We may assume as above that $c$ intersects $N$ transversely, and hence $c$ meets $N$ at finite points, $y_{1}=c\left(s_{1}\right), \cdots, y_{r}=c\left(s_{r}\right), 0<s_{1} \cdots<s_{r}$ $<1$. We shall construct by induction on $r$ a path $v$ from $p_{0}$ to $p_{1}$ such that $v$ does not intersect $N$. Let $u$ be a closed path such that $u$ intersects $N$ at a single point $y_{0}=u\left(t_{0}\right)$ for $t_{0} \in(0,1)$. There is a path $d$ from $y_{1}$ to $y_{0}$ in $N$. Let $\varepsilon>0$ be sufficiently small. Then, there is a path $d_{-}$in $M-N$ from $c\left(s_{1}-\varepsilon\right)$ to $u\left(t_{0}-\delta\right)$ along $d$, where $\delta$ is a positive or negative real number with a sufficiently small absolute value. Similarly, there is $d_{+}$from $c\left(s_{1}+\varepsilon\right)$ to $u\left(t_{0}+\delta\right) . \quad c\left(\left[0, s_{1}-\varepsilon\right]\right) \cup$ (image $d_{-}$) 
$\cup u\left(I-\left(t_{0}-\delta, t_{0}+\delta\right)\right) \cup$ (image $\left.d_{+}\right) \cup c\left(\left[s_{1}+\varepsilon, 1\right]\right)$ is an image of a path $c^{\prime}$ from $p_{0}$ to $p_{1}$ which intersects $N$ at $(r-1)$ points. Then $c^{\prime}$ has the inductive property. This proves Lemma 2 .

Let $H: H_{1}(M ; Z) \rightarrow Z_{(1)}+\cdots+Z_{(m)}$ be an epimorphism onto a free abelian group of $\operatorname{rank} m, Z_{(i)} \cong Z(i=1, \cdots, m)$. Let $p_{i}: Z_{(1)}+\cdots+Z_{(m)}$ $\rightarrow Z_{(i)}$ be the projection onto the $i$-th factor. By Künneth's theorem the map $\kappa: H^{1}(M ; Z) \rightarrow \operatorname{Hom}\left(H_{1}(M ; Z), Z\right)$ induced from slant operation is an isomorphism since $H_{0}(M ; Z)$ is free abelian. Assume $\partial M=\phi$. Let $\delta: H^{1}(M ; Z) \rightarrow H_{n-1}(M ; Z)$ be the Poincaré duality isomorphism, and let $\theta_{i}=\delta \kappa^{-1}\left(p_{i} H\right)$. For $\gamma \in H_{1}(M ; Z)$,

$$
\begin{aligned}
& \gamma \cdot \theta_{i}=\gamma \cap \delta^{-1}\left(\theta_{i}\right) \\
& =\gamma \cap\left(\kappa^{-1}\left(p_{i} H\right)\right) \\
& =p_{i} H(\gamma),
\end{aligned}
$$

where $\cap$ denotes cup product. Thus we have

$$
\gamma \cdot \theta_{i}=p_{i} H(\gamma) \quad \text { for } \gamma \in H_{1}(M ; Z) .
$$

Now, we set the following result of Nakatsuka.

LEMMA 3 ([3]). Let $M$ be a compact connected orientable manifold of dimension $n \geqq 3$ and $\theta \in H_{n-1}(M ; Z)$. Then, there is a connected orientable $(n-1)$-submanifold $N$ in $M$ such that $\theta=[N]$ if and only if there is a homology class $\gamma \in H_{1}(M ; Z)$ such that the intersection number $\gamma \cdot \theta=1$.

Proposition 1. Let $M$ be a connected orientable closed manifold of dimension $n \geqq 3$, and let $H: H_{1}(M ; Z) \rightarrow Z_{(1)}+\cdots+Z_{(m)}$ be an epimorphism. Then, there are connected closed codimension one submanifolds $N_{1}, \cdots, N_{m}$ of $M$ satisfying the followings.

(i) $N_{1}, \cdots, N_{m}$ are in general position in $M$.

(ii) $\gamma \cdot\left[N_{i}\right]=p_{i} H(\gamma)$ for any $\gamma \in H_{1}(M ; Z), i=1, \cdots, m$.

(iii) $N_{i}-N_{1} \cup \cdots \cup N_{i-1}$ is connected for $i=2, \cdots, m$.

(iv) $M-N_{1} \cup \cdots \cup N_{m}$ is connected.

(v) $H j_{*}\left(H_{1}\left(M-N_{1} \cup \cdots \cup N_{i} ; Z\right)\right)=Z_{(i+1)}+\cdots+Z_{(m)}$ for $i=1$, $\cdots, m-1$, and $=0$ for $i=m$. Here, $j$ is the inclusion $M-N_{1} \cup \cdots$ $\cup N_{i} \rightarrow M$.

Proof. Since $p_{i} H: H_{1}(M ; Z) \rightarrow Z_{(i)}$ is an epimorphism, there is $\gamma_{i}$ $\in H_{1}(M ; Z)$ such that $H\left(\gamma_{i}\right)$ is the generator of $Z_{(i)}, i=1, \cdots, m$. Then, 
by Lemma $3, \gamma_{i} \cdot \theta_{i}=p_{i} H\left(\gamma_{i}\right)=1$ implies that there are connected orientable closed $(n-1)$-submanifolds $N_{1}^{\prime}, \cdots, N_{m}^{\prime}$ in $M$ such that $\left[N_{i}^{\prime}\right]=\theta_{i}$, $i=1, \cdots, m$. $N_{1}^{\prime}, \cdots, N_{m}^{\prime}$ may be assumed to be in general position.

We vary $N_{i}^{\prime}$ to $N_{i}, i=1, \cdots, m$, by induction on $i$ so that $N_{1}, \cdots, N_{i}$ satisfy the following condition $C(\mathrm{i})$. Denote $M_{i}=M-N_{1} \cup \cdots \cup N_{i}$.

$C$ (i) (i ) $N_{1}, \cdots, N_{i}$ are in general position in $M$.

(ii) $\left[N_{k}\right]=\theta_{k}, k=1, \cdots, i$.

(iii) $N_{k}-N_{1} \cup \cdots \cup N_{k-1}$ is connected for $k=2, \cdots, i$ if $i \geqq 2$.

(iv) $M_{i}$ is connected.

(v) $H \circ j_{*}\left(H_{1}\left(M_{k} ; Z\right)=Z_{(k+1)}+\cdots+Z_{(m)}\right.$ for $k=1, \cdots, i$.

First, we construct $N_{1}$ as follows. Since $n \geqq 3$, there are simple closed paths $c_{2}, \cdots, c_{m}$ such that $\left[c_{2}\right]=\gamma_{2}, \cdots,\left[c_{m}\right]=\gamma_{m}$ and that they are mutually disjoint. By Lemma 1 , there is a manifold $N_{1}$ such that $\left[N_{1}\right]=\left[N_{1}^{\prime}\right]$ and that $N_{1}$ does not intersects $c_{2}, \cdots, c_{m}$. By Lemma 2 , the existence of $\gamma_{1}$ implies that $M-N_{1}$ is connected. Since $c_{2}, \cdots, c_{m}$ are contained in $M_{1}$ and $0=\gamma \cdot\left[N_{1}\right]=p_{1} H(\gamma)$ for $\gamma \in H_{1}\left(M_{1} ; Z\right)$, it is not difficult to see that $H j_{*}\left(H_{1}\left(M_{1} ; Z\right)\right)=Z_{(2)}+\cdots+Z_{(m)}$. Then, $N_{1}$ satisfies the condition $C(1)$.

Next, suppose that $N_{1}, \cdots, N_{i}$ are constructed so that the condition $C(\mathrm{i})$ is satisfied. Now, we construct $N_{i+1}$ so that $N_{1}, \cdots, N_{i}, N_{i+1}$ satisfy $C(i+1)$. By (v) of $C(\mathrm{i})$ there is a simple closed path $c_{i+1}$ in $M_{i}$ realizing $\gamma_{i+1} \in H_{1}(M ; Z)$, and hence the intersection number $c_{i+1} \cdot\left(N_{i+1}^{\prime}-N_{1}\right.$ $\left.\cup \cdots \cup N_{i}\right)=c_{i+1} \cdot N_{i+1}^{\prime}=\left[c_{i+1}\right] \cdot\left[N_{i+1}^{\prime}\right]=\gamma_{i+1} \cdot \theta_{i+1}$ is 1 . We can take $c_{i+1}$ so that it intersects $N_{i+1}^{\prime}$ transversely. Then, by the method of the proof of Lemma 3 in [3], there is a closed manifold $N_{i+1}^{\prime \prime}$ such that (i) $N_{i+1}^{\prime \prime} \cap M_{i}$, so $N_{i+1}^{\prime \prime}$, is connected and (ii) $\left[N_{i+1}^{\prime \prime}\right]=\left[N_{i+1}^{\prime}\right]$ in $H_{n-1}(M ; Z)$ and $\left[N_{i+1}^{\prime} \cap M_{i}\right]=\left[N_{i+1}^{\prime} \cap M_{i}\right]$ in $H_{n-1}\left(M_{i} ; Z\right)$. Here, $N_{i+1}^{\prime \prime}$ is obtained by attaching slender 1-handles to $N_{i+1}^{\prime}$ along simple arcs in $M_{i}$. Next, we vary $N_{i+1}^{\prime \prime}$ to construct $N_{i+1}$ so that $N_{1}, \cdots, N_{i}$ and $N_{i+1}$ satisfy the condition $C(i+1)$. By (v) of $C(\mathrm{i})$, there are simple closed paths $c_{i+2}, \cdots, c_{m}$ in $M_{i}$ realizing $\gamma_{i+2}, \cdots, \gamma_{m}$, respectively. We may assume that they intersect $N_{i+1}^{\prime \prime}$ transversely and that they are mutually disjoint. Similarly as the construction of $N_{1}$, we obtain $N_{i+1}$ from $N_{i+1}^{\prime \prime}$ by attaching slender 1-handles along simple arcs contained in $c_{i+2}, \cdots, c_{m}$ so that $N_{i+1}$ does not intersects $c_{i+2}, \cdots, c_{m}$, that $\left[N_{i+1}\right]=\left[N_{i+1}^{\prime}\right]$, and that $H \circ j_{*}\left(H_{1}\left(M-N_{1}\right.\right.$ $\left.\left.\cup \cdots \cup N_{i} \cup N_{i+1} ; Z\right)\right)=Z_{(i+2)}+\cdots+Z_{(m)}$. Since $c_{i+1}$ is a path in $M_{i}$ and $c_{i+1} \cdot\left(N_{i+1} \cap M_{i}\right)=c_{i+1} \cdot N_{i+1}=1$, Lemma 2 implies that $M_{i+1}=M_{i}$ 
$-N_{i+1}$ is connected. From the above, we can see that $N_{1}, \cdots, N_{i+1}$ satisfy the condition $C(i+1)$. This proves Proposition 1 .

\section{§3. Proof of Theorem 1}

Let $\mathscr{F}$ be a codimension one foliation of class $C^{r}$ of an orientable $(n+1)$-manifold $M$, and suppose that an orientable $n$-manifold $F_{0}$ is a closed leaf of $\mathscr{F}$. Let $\nu: U \rightarrow F_{0}$ is an $R$-bundle of a bicollar $U$ of $F_{0}$, and let $\nu_{+}: U_{+} \rightarrow F_{0}$ is an $\boldsymbol{R}_{+}$-bundle of a collar $U_{+}$of $F_{0}, \boldsymbol{R}=(-\infty, \infty)$ and $R_{+}=[0, \infty) . \quad F_{0}$ is identified with the zero section of $\nu$ or $\nu_{+}$, and the fibres of $\nu$ and $\nu_{+}$are identified with $\boldsymbol{R}$ and $\boldsymbol{R}_{+}$respectively.

A curve $u:[0,1] \rightarrow U$ is called a leaf curve from $u(0)$ to $u(1)$ if the image of $u$ is contained in a leaf. Let $y \in \nu^{-1} u(0)$ and let $u_{y}:[0,1] \rightarrow U$ be a leaf curve such that $u_{y}(0)=y$ and $\nu u_{y}(t)=u(t)$ for any $t \in[0,1]$. We call $u_{y}$ the $y$-lift of $u$. There exists at most one $y$-lift of $u$. If there is the $y$-lift of $u$ for any $y$ in $\left[y_{1}, y_{2}\right] \subset \boldsymbol{R}=\nu^{-1} u(0)$ the holonomy map $h_{u}$ from $\left[y_{1}, y_{2}\right]$ into $\boldsymbol{R}=\nu^{-1} u(b)$ is defined by $h_{u}(y)=u_{y}(b)$.

Let $x_{*} \in F_{0}$ and $u$ be a closed leaf curve with base point $x_{*}$. The germ of $h_{u}$ at 0 is called the holonomy of $u$. The holonomy of $u$ is determined by the homotopy class $[u]$ of $u$ in $\pi_{1}\left(F_{0}, x_{*}\right)$ and is independent of the choice of $\nu$ up to conjugations by origin preserving diffeomorphism of $\boldsymbol{R}$. Let $G^{r}$ be the group of the germs at 0 of all orientation-preserving local $C^{r}$-diffeomorphisms of $\boldsymbol{R}$ which leave the origin fixed. A homomorphism $h: \pi_{1}\left(F_{0}, x_{*}\right) \rightarrow G^{r}$ is defined by corresponding the holonomy of $u$ to $[u] \in \pi_{1}\left(F_{0}, x_{*}\right)$. The image of the homomorphism $h$ is called the holonomy group of $F_{0}$ and denoted by $\Phi\left(F_{0}\right)$. The one-sided holonomy group $\Phi_{+}\left(F_{0}\right)$ of $F_{0}$ is defined similarly by replacing $\nu$ and $R$ by $\nu_{+}$and $\boldsymbol{R}_{+}$.

A proof of the following Lemma 4 is found in the proof of Lemma 2 in [4].

LEMMA 4. If $\Phi_{+}\left(F_{0}\right)$ is the trivial group there is a neighborhood $U_{0}$ of $F_{0}$ in $U_{+}$such that the restricted foliation $\mathscr{F} \mid U_{0}$ is trivial; i.e. for each leaf $F$ of $\mathscr{F} \mid U_{0}, \nu: F \rightarrow F_{0}$ is a diffeomorphism.

In this paper, we assume that $\Phi_{+}\left(F_{0}\right)$ is abelian, then $\Phi_{+}\left(F_{0}\right)$ is free abelian since $G^{r}$ has no torsion element. Let $\iota: \Phi_{+}\left(F_{0}\right) \rightarrow Z_{(1)}+\cdots+Z_{(m)}$ be an isomorphism and let $\eta: \pi_{1}\left(F_{0}, x_{*}\right) \rightarrow H_{1}\left(F_{0}: Z\right)$ be the Hurewicz homomorphism. Then, there is an epimorphism $H: H_{1}\left(F_{0} ; Z\right) \rightarrow Z_{(1)}+\cdots$ 
$+Z_{(m)}$ such that $H \eta=c h$. Let $p_{i}$ be the projection from $Z_{(1)}+\cdots+Z_{(m)}$ onto the $i$-th factor. Thus we have the following diagram.

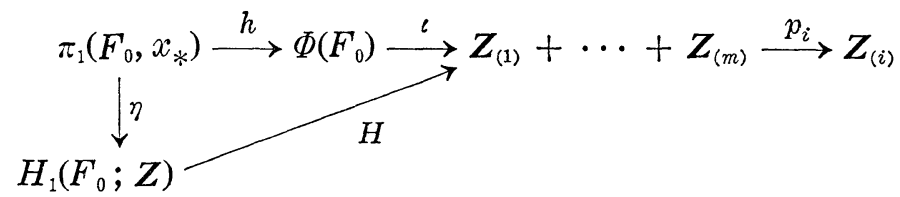

Let $N_{1}, \cdots, N_{m}$ be codimension one smooth submanifolds in $F_{0}$ such that they are in the general position and that $F_{0}-N_{1} \cup \cdots \cup N_{m}$ is connected. Denote by $F_{1}$ the compact manifold with boundary obtained by attaching two copies $N_{1}^{\prime}$ and $N_{1}^{\prime \prime}$ of $N_{1}$ to $F_{0}-N_{1}$, so that $\partial F_{1}=N_{1}^{\prime}$ $\cup N_{1}^{\prime \prime}$. Then, a local diffeomorphism $g_{1}: F_{1} \rightarrow F_{0}$ is defined by $g_{1}(x)=x$ for $x \notin \partial F_{1}$ and $g_{1}\left(y^{\prime}\right)=g_{1}\left(y^{\prime \prime}\right)=y$ for $y \in N_{1}$, where $y^{\prime} \in N_{1}^{\prime}$ and $y^{\prime \prime} \in N_{1}^{\prime \prime}$ are the copies of $y \in N_{1} . \quad g_{1}^{-1}\left(N_{i}\right) \subset F_{1}$ is denoted also by $N_{i}, i=2, \cdots, m$. Inductively we define $F_{2}, \cdots, F_{m}$ and $g_{i}: F_{i} \rightarrow F_{i-1}, i=2, \cdots, m$, similarly as above. The boundaries of $F_{2}, \cdots, F_{m}$ have possibly corners. Let $g: F_{m} \rightarrow F_{0}$ be the composition $g_{j} \cdots g_{1} . \quad F_{m}$ is said to be the manifold which is obtained by cutting open $F_{0}$ along $N_{1}, \cdots, N_{m}$. $g$ is said to be the map pasting $F_{m}$ on $F_{0}$.

Proof of Theorem 1. If $n=1$, this theorem is well known in the theory of dynamical system. If $n>2$, let $N_{1}, \cdots, N_{m}$ be the manifolds obtained by Proposition 1 for the epimorphism $H: H_{1}\left(F_{0} ; Z\right) \rightarrow Z_{(1)}+\cdots$ $+Z_{(m)} \cong \Phi_{+}\left(F_{0}\right)$ defined above. If $n=2$, let $p$ be the genus of $F_{0}$. Then we can take simple closed curves $N_{1}, \cdots, N_{2 p}$ in $F_{0}$ such that $N_{i} \cap N_{j}$ is at most one point for any different $i, j$ and that $F_{0}-N_{1} \cup \cdots \cup N_{2 p}$ is an open 2-ball. We define $N_{1}, \cdots, N_{\ell}$ in the theorem as above.

Since $F_{0}-N_{1} \cup \cdots \cup N_{\ell}$ is a 2-ball for $n=2, \Phi_{+}\left(F_{0}-N_{1} \cup \cdots \cup N_{\ell}\right)$ $=0$ in $\mathscr{F} \mid \nu_{+}^{-1}\left(F_{0}-N_{1} \cup \cdots \cup N_{\ell}\right)$. When $n>2$, let $c$ be a simple closed path in $F_{0}-N_{1} \cup \cdots \cup N_{m}$. Let $\gamma$ be the homotopy class of $c$ in $\pi_{1}\left(F_{0}, x_{*}\right)$, $x_{*} \in F_{0}-N_{1} \cup \cdots \cup N_{m}$, and $\left[N_{i}\right]$ be the homology class of $N_{i}$ in $H_{1}\left(F_{0} ; Z\right)$. Then, by Proposition 1,

$$
\begin{aligned}
p_{i} \iota h(\gamma) & =p_{i} H_{\eta}(\gamma), \\
& =p_{i} H([c]), \quad[c] \in H_{1}\left(F_{0} ; Z\right) \\
& =[c] \cdot\left[N_{i}\right]=0
\end{aligned}
$$

since $c \cap N_{i}=\phi$, for $i=1, \cdots, m$. This implies $\Phi_{+}\left(F_{0}-N_{1} \cup \cdots \cup N_{\ell}\right)$ $=0$, if $n>2$. By using Lemma 4 , wee see that there is a injective 
$C^{r}$-diffeomorphism $\xi:\left(F_{0}-N_{1} \cup \cdots \cup N_{\ell}\right) \times[0, \delta] \rightarrow U_{+}$such that (i) $\xi$ maps each $\left(F_{0}-N_{1} \cup \cdots \cup N_{\ell}\right) \times\{t\}$ into a leaf of $\mathscr{F} \mid U_{+}$and that (ii) $\nu_{+} \xi(x, t)=x$ for $x \in F_{0}-N_{1} \cup \cdots \cup N_{\ell}$ and $t \in[0, \delta]$. Put $\xi\left(\left(F_{0}-N_{1}\right.\right.$ $\left.\left.\cup \cdots \cup N_{\ell}\right) \times[0, \delta]\right)=\tilde{F}_{*} \subset \nu_{+}^{-1}\left(F_{0}-N_{1} \cup \cdots \cup N_{\ell}\right)$. By identifying $\xi(x, t)$ with $(x, t), \quad(x, t) \in\left(F_{0}-N_{1} \cup \cdots \cup N_{\ell}\right) \times[0, \delta]$ is a coordinates of $\tilde{F}_{*}$. Putting $V^{\prime}=\operatorname{cl} F_{*}, V^{\prime}$ is a closed neighborhood of $F_{0}$ in $U_{+}$. We are dealing with the holonomy maps and the holonomies for closed paths in $F_{0}$ with the fixed base point $x_{*} \in \operatorname{int} \tilde{F}_{*}$. From now on in this section a holonomy maps are considered as local diffeomorphisms of $[0, \delta]$ by identifying $[0, \delta]$ with $x_{*} \times[0, \delta]$, where $x_{*} \times[0, \delta]$ is the expression of the above coordinates.

The number of the connected components of $N_{i}-N_{1} \cup \cdots \cup N_{i-1}$ $\cup N_{i+1} \cup \cdots \cup N_{\ell}$ is only one if $n=2$. For $n>3$, let $N_{i j}$ be one of these components. For any $x$ in $N_{i j}$ there is a closed path $v_{x}$ in $F_{0}$ with base point $x_{*}$ such that $v_{x}$ intersects $N_{1} \cup \cdots \cup N_{\ell}$ at only one point $x$, since $F_{0}-N_{1} \cup \cdots \cup N_{\ell}$ is connected. There is $\varepsilon_{x}$ with $0<\varepsilon_{x}$ $\leqq \delta$ such that there is a leaf curve of $\mathscr{F} \mid V^{\prime}$ which is the lift of $v_{x}$ starting from $\left(x_{*}, \varepsilon_{x}\right) \in v_{+}^{-1}\left(x_{*}\right)$. So, the holonomy map $f_{x}$ of $v_{x}$ is defined on $\left[0, \varepsilon_{x}\right]$. Let $\tilde{v}_{x}$ be a lift of $v_{x}$ and let $\tilde{v}_{x}(0)=s^{\prime}, v_{x}(1)=s^{\prime \prime}$ in $\left\{x_{*}\right\}$ $\times\left[0, s_{x}\right] \subset v_{+}^{-1}\left(x_{*}\right)$. Let $\tilde{v}_{x}\left(t_{0}\right) \in v_{+}^{-1}(x)$. For any $t^{\prime}, t^{\prime \prime}$ with $0 \leqq t^{\prime}<t_{0}<t^{\prime \prime}$ $\leqq 1$, we have $\tilde{v}_{x}\left(t^{\prime}\right)=\left(v_{x}\left(t^{\prime}\right), s^{\prime}\right)$ and $\tilde{v}_{x}\left(t^{\prime \prime}\right)=\left(v_{x}\left(t^{\prime \prime}\right), s^{\prime \prime}\right)$ in the coordinates $\tilde{F}_{*}=\left(F_{0}-N_{1} \cup \cdots \cup N_{\ell}\right) \times[0, \delta]$, since $\mathscr{F} \mid \tilde{F}_{*}$ is trivial. Hence, we have $f_{x}\left(s^{\prime}\right)=s^{\prime \prime}$. Let $N_{i j}$ have the orientation which is compatible with the inclusion $N_{i j} \subset N_{i}$ and the given orientation of $N_{i}$. For another point $y$ in $N_{i j}$ let $v_{y}$ be a closed curve as above such that $\left[v_{y}\right] \cdot\left[N_{i j}\right]$ $=\left[v_{x}\right] \cdot\left[N_{i j}\right]$. From the triviality of $\mathscr{F} \mid \tilde{F}_{*}$ it is easy to see that the source of the holonomy map $f_{y}$ of $v_{y}$ is same as $f_{x}$ and that $f_{y}=f_{x}$ on it, i.e. $f_{y}(s)=f_{x}(s)$ for any $s \in\left[0, \varepsilon_{x}\right]$. Therefore, there are $\varepsilon_{i j}$ with $0<\varepsilon_{i j}<\delta$ and an injective diffeomorphism $f_{i j}:\left[0, \varepsilon_{i j}\right] \rightarrow[0, \delta]$ satisfying the following property; for any $x$ in $N_{i j}$ and any closed path $v_{x}$ in $F_{0}$ with base point $x_{*}$ such that $v_{x}$ intersects $N_{1} \cup \cdots \cup N_{\ell}$ at only one point $x$ and that $\left[v_{x}\right] \cdot\left[N_{i}\right]=1$, the holonomy map of $v_{x}$ is defined on $\left[0, \varepsilon_{i j}\right]$ and is equal to $f_{i j}$. For two components $N_{i j}$ and $N_{i k}$ of $N_{i}-N_{1}$ $\cup \cdots \cup N_{i-1} \cup N_{i+1} \cup \cdots \cup N_{\ell}$ the holonomy maps $f_{i j}$ and $f_{i k}$ are coincide on a small neighborhood of 0 , since $\left[v_{x}\right] \cdot\left[N_{i}\right]=\left[v_{y}\right] \cdot\left[N_{i}\right]=1$ so the holonomies of $v_{x}$ and $v_{y}$ are coincide. Hence, there are $\varepsilon_{i}$ with $0<\varepsilon_{i}$ $<\delta$ and an injective diffeomorphism $f_{i}:\left[0, \varepsilon_{i}\right] \rightarrow[0, \delta]$ satisfying the same 
property as above. Therefore, there are $0<\varepsilon<\delta$ and injective diffeomorphisms $f_{1}, \cdots, f_{\ell}$ for $N_{1}, \cdots, N_{\ell}$ satisfying the following property; for any $x$ in $N_{i}$ and any closed path $v_{x}$ in $F_{0}$ with base point $x_{*}$ such that $v_{x}$ intersects $N_{1} \cup \cdots \cup N_{\ell}$ at only one point $x$ and that $\left[v_{x}\right] \cdot\left[N_{i}\right]$ $=1$, the holonomy map of $v_{x}$ is defined on $[0, \varepsilon]$ and is equal to $f_{i}$. Since $\Phi\left(F_{0}\right)$ is abelian, we may assume that $f_{1}, \cdots, f_{\ell}$ are mutually commutative by choosing $\varepsilon$ sufficiently small.

Since $f_{i}$ and $f_{i}^{-1}$ are monotonously increasing, $f_{i}(\varepsilon)>\varepsilon$ implies $\varepsilon>f_{i}^{-1}(\varepsilon)$. So, replacing $f_{i}$ by $f_{i}^{-1}$ (i.e. replacing the orientation of $N_{i}$ ) if necessary, we can suppose that $\varepsilon \geqq f_{i}(\varepsilon)$ for all $i$. Notice that $N_{i}=\bigcup_{j} \operatorname{cl} N_{i j}$ and $g^{-1}\left(N_{i j}\right)=N_{i j}^{\prime} \cup N_{i j}^{\prime \prime}$. Here, $g: F_{*} \rightarrow F_{0}$ is the diffeomorphism pasting $F_{*}$ on $F_{0}, F_{*}$ is the manifold obtained by cutting open $F_{0}$ along $N_{1}, \cdots, N_{\ell}$, and $N_{i j}^{\prime}, N_{i j}^{\prime \prime}$ are diffeomorphic manifolds such that $g\left(N_{i j}^{\prime}\right)$ $=N_{i j}=g\left(N_{i j}^{\prime \prime}\right)$. Then, $g^{-1}\left(N_{i}\right)=N_{i}^{\prime} \cup N_{i}^{\prime \prime}$, where $N_{i}^{\prime}$ and $N_{i}^{\prime \prime}$ are diffeomorphic manifolds such that $N_{i}^{\prime}=\bigcup_{j} \operatorname{cl} N_{i j}^{\prime}, N_{i}^{\prime \prime}=\bigcup_{j} \operatorname{cl} N_{i j}^{\prime \prime}$ and $g\left(N_{i}^{\prime}\right)=N_{i}$ $=g\left(N_{i}^{\prime \prime}\right)$. Since $N_{1}, \cdots, N_{\ell}$ are in general position and $f_{1}, \cdots, f_{\ell}$ are mutually commutative, it is not difficult to show that a quotient manifold $X_{f}$ is well defined from $F_{*} \times[0, \varepsilon]$ by identifying $(x, s) \in N_{i}^{\prime} \times[0, \varepsilon]$ and $\left(x, f_{i}(s)\right) \in N_{i}^{\prime \prime} \times[0, \varepsilon]$. Let $\mathscr{F}_{f}$ be the foliation on $X_{f}$ induced from the trivial foliation of $F_{*} \times[0, \varepsilon]$. Since $\operatorname{int} F_{*}$ is diffeomorphic to $F_{0}-N_{1}$ $\cup \cdots \cup N_{\ell}$, we can see from the above facts that there is a $C^{r}$-diffeomorphism from a neighborhood $V$ of $F_{0}$ in $U_{+}$onto $X_{f}$ mapping each leaf of $\mathscr{F} \mid V$ onto a leaf of $\mathscr{F}_{f}$.

By the constructions of $f_{1}, \cdots, f_{\ell}$, these maps satisfies the property (ii)-(c) in the theorem. This completes the proof of Theorem 1.

\section{§4. Proof of Theorem 2}

LEMma 5. Let $f_{1}, \cdots, f_{\ell}$ be injective homeomorphisms from $[0, \varepsilon]$ into $[0, \varepsilon]$ such that $f_{i}(0)=0$ for $i=1, \cdots, \ell$. Suppose

$$
f_{i} f_{j}(t)=f_{j} f_{i}(t), \quad i, j=1, \cdots, \ell .
$$

Put

$$
\begin{array}{ll}
h_{1}(t)=f_{i_{\alpha}}^{\sigma_{\alpha}} \cdots f_{i_{1}}^{\sigma_{1}}(t), & \sigma_{a}= \pm 1, \\
h_{2}(t)=f_{j_{\beta}}^{\tau_{\beta}} \cdots f_{j_{1}}^{\tau_{1}}(t), & \tau_{b}= \pm 1 .
\end{array}
$$

Then $h_{1}(t)=h_{2}(t)$ for any $t$ such that $h_{1}(t)$ and $h_{2}(t)$ are defined if 
(3) $\sum_{i_{a}=i} \sigma_{i_{a}}=\sum_{j_{b}=i} \tau_{j_{b}}, \quad i=1, \cdots, \ell, \quad a=1, \cdots, \alpha, \quad b=1, \cdots, \beta$.

Here, $f_{i}^{-1}$ is considered to be defined on $\left[0, f_{i}(\varepsilon)\right]$.

Proof. By the assumption we have

$$
f_{i}^{\sigma} f_{j}^{\tau}(t)=f_{j}^{\tau} f_{i}^{\sigma}(t), \quad \sigma, \tau= \pm 1, i, j=1, \cdots, \ell
$$

for any $t$ such that both sides of the expression are defined. We define a linear order $<$ in the set $\left\{f_{1}, \cdots, f_{\ell}, f_{1}^{-1}, \cdots, f_{\ell}^{-1}\right\}$ as follows; for $f_{i}$, $f_{j}$ and $f_{i}^{-1}, f_{j}^{-1}$, we define $f_{i}<f_{j}$ and $f_{i}^{-1}<f_{j}^{-1}$ respectively if $i<j$, and we define $f_{i}<f_{j}^{-1}$ for any $f_{i}$ and $f_{j}^{-1}$. It is not difficult to see that $<$ is a linear order.

Next, we show that if $f_{i}^{\sigma_{i}} f_{j}^{\sigma_{j}}(t)$ is defined and $f_{i}^{\sigma_{i}}<f_{j}^{\sigma_{j}}, f_{j}^{\sigma_{j}} f_{i}^{\sigma_{i}}(t)$ is also defined and $f_{i}^{\sigma_{i}} f_{j}^{\sigma_{j}}(t)=f_{j}^{\sigma_{j}} f_{i}^{\sigma_{i}}(t)$. This property is trivial for $f_{i}$ and $f_{j}$. For $f_{i}^{-1}$ and $f_{j}^{-1}$ it is shown as follows. Suppose $f_{i}^{-1}<f_{j}^{-1}$. If $f_{i}^{-1} f_{j}^{-1}(t)$ is defined, $f_{j}^{-1}(t) \leqq f_{i}(\varepsilon)$, so $t \leqq f_{j} f_{i}(\varepsilon)$. Since $f_{j} f_{i}(\varepsilon)=f_{i} f_{j}(\varepsilon)$, $t \leqq f_{i} f_{j}(\varepsilon)$. Hence, $f_{i}^{-1}(t) \leqq f_{j}(\varepsilon)$, and so $f_{j}^{-1} f_{i}^{-1}(t)$ is defined. Then $f_{i}^{-1} f_{j}^{-1}(t)=f_{j}^{-1} f_{i}^{-1}(t)$. Finally, for $f_{i}$ and $f_{j}^{-1}$ it is shown as follows. Suppose $f_{i}<f_{j}^{-1}$. If $f_{i} f_{j}^{-1}(t)$ is defined, $t \leqq f_{j}(\varepsilon)$, so $t_{i}(t) \leqq f_{i} f_{j}(\varepsilon)$. Since $f_{i} f_{j}(\varepsilon)=f_{j} f_{i}(\varepsilon) \leqq f_{j}(\varepsilon), f_{i}(t) \leqq f_{j}(\varepsilon)$. Then, $f_{j}^{-1} f_{i}(t)$ is defined, and so $f_{i} f_{j}^{-1}(t)=f_{j}^{-1} f_{i}(t)$.

If $f_{j}(t)$ or $f_{j}^{-1}(t)$ is defined, $f_{j}\left(f_{i}^{-1} f_{i}\right)(t)$ or $\left(f_{i}^{-1} f_{i}\right) f_{j}^{-1}(t)$ is defined and $f_{j}(t)=f_{j}\left(f_{i}^{-1} f_{i}\right)(t)$ or $f_{j}^{-1}(t)=\left(f_{i}^{-1} f_{i}\right) f_{j}^{-1}(t)$, respectively. Next, we interplate $f_{i}^{-1} f_{i}$ in the right hand of the expressions of (1) and (2) if necessary so that the same number of $f_{i}$ and $f_{i}^{-1}$ are contained in these expressions for each $i=1, \cdots, \ell$. Finally, we change the order in the rows of the terms in these expressions to the order induced from $<$. Then, the obtained expressions are identical. This proves $h_{1}(t)=h_{2}(t)$.

LEMMA 6. Let $\mathscr{F}$ be a transversely orientable $C^{r}$-foliation of codimension one, $r \geqq 1$, and let $F_{0}$ be a compact leaf of $\mathscr{F}$. Let $\nu$ be a normal $\boldsymbol{R}_{+}$-bundle map from a collar $U_{+}$onto $F_{0}$ such that $\nu$ is transverse to $\mathscr{F}$, and let $F \in \mathscr{F}$ be asymptotic to $F_{0}$ in $U_{+}$. Then, the following properties are satisfied.

(i) For a neighborhood $V$ of $F_{0}$ in $U_{+}$, let $F_{V}$ be an asymptotic leaf of $\mathscr{F} \mid V$ to $F_{0}$ such that $F_{V} \cap F \neq \phi$. Then, an unique regular covering $\tilde{\nu}: \tilde{F} \rightarrow F_{0}$ is associated with $F_{V}$ and $\nu_{*}\left(\pi_{1}\left(F_{V}\right)\right)=\tilde{\nu}_{*}\left(\pi_{1}(\tilde{F})\right)$ in $\pi_{1}\left(F_{0}\right)$ if and only if the following condition $(*)$ is satisfied.

(*) For a point $x_{*}$ in $F_{0}$ and any closed path $u$ in $F_{0}$ with the base 
points $x_{*}$ let $y$ and $z$ be any two points in $\nu^{-1}\left(x_{*}\right) \cap F_{V}$ such that $h_{u}(y)$ and $h_{u}(z)$ are defined, where $h_{u}$ is the holonomy map of $u$. Then, $h_{u}(y)$ $=y$ if and only if $h_{u}(z)=z$.

(ii) Suppose $F$ and $V$ satisfies $(*)$. Then, for any neighborhood $V^{\prime}$ of $F_{0}$ in $V$, the same regular covering as $\tilde{\nu}$ is associated with $F_{V /}$.

Proof. Let $\tilde{\nu}: \tilde{F} \rightarrow F_{0}$ be a regular covering and let $u$ be a closed curve in $F_{0}$ with base point $x_{*}$. For $y$ and $z$ in $\tilde{\nu}^{-1}\left(x_{*}\right)$ let $u_{y}$ and $u_{z}$ be the lifts of $u$ starting from $y$ and $z$ respectively. Then, $u_{y}$ is a closed curve if and only if $u_{z}$ is so. Therefore, if there is an associated regular covering with $F_{V}$, condition $(*)$ is satisfied.

Next, we prove the converse. Define a subgroup $G\left(F_{V}\right)$ of $\pi_{1}\left(F_{0}, x_{*}\right)$ by

$$
\begin{array}{r}
G\left(F_{V}\right)=\left\{\alpha \in \pi_{1}\left(F_{0}, x_{*}\right) \mid \text { there is a closed curve } \bar{u} \text { in } F_{V}\right. \\
\text { such that }[\nu \bar{u}]=\alpha\},
\end{array}
$$

where [ ] denotes the homotopy class. We must show that $G\left(F_{V}\right)$ is a subgroup of $\pi_{1}\left(F_{0}, x_{*}\right)$. For $\alpha$ and $\beta$ in $G\left(F_{V}\right)$ there are closed curves $\bar{u}$ and $\bar{v}$ in $F_{V}$ such that $[\nu \bar{u}]=\alpha$ and $[\nu \bar{v}]=\beta^{-1}$. Let $y, z \in \nu^{-1}\left(x_{*}\right)$ be the base point of $\bar{u}, \bar{v}$. Assume $x_{*}<y<z$ in the line $\nu^{-1}\left(x_{*}\right)$. Put $\nu \bar{u}=u$ and $\nu \bar{v}=v$. By the existence of $\bar{v}, h_{v}(y)$ is defined. Condition (*) implies $h_{v}(y)=y$. So, there is the lift $\tilde{v}$ of $v$ starting from $y$. $\tilde{v}$ is a closed curve in $F_{V}$. Then, $\bar{u} \tilde{v}$ is a closed curve in $F_{V}$ such that $[\nu(\bar{u} \tilde{v})]$ $=\alpha \beta^{-1}$. Therefore, $\alpha \beta^{-1} \in G\left(F_{V}\right)$.

To the conjugacy class of a subgroup of $\pi_{1}\left(F_{0}, x_{*}\right)$ an unique covering of $F_{0}$ exists. Let $\tilde{\nu}: \tilde{F} \rightarrow F_{0}$ be the covering corresponding to the conjugacy class including $G\left(F_{V}\right)$. Then, for $\tilde{y} \in \tilde{\nu}^{-1}\left(x_{*}\right), \tilde{\nu}_{*}\left(\pi_{1}(F, y)\right)$ is a subgroup of $\pi_{1}\left(F_{0}, x_{*}\right)$ which is conjugate to $G\left(F_{V}\right)$.

Next, we define the map $i: F_{V} \rightarrow \tilde{F}$. Fix two points $y_{*} \in F_{V}$ and $\tilde{y}_{*} \in \tilde{F}$ so that $\nu\left(y_{*}\right)=\tilde{\nu}\left(\tilde{y}_{*}\right)=x_{*}$ and that $\tilde{\nu}_{*}\left(\pi_{1}\left(\tilde{F}, \tilde{y}_{*}\right)\right)=G\left(F_{V}\right)$. For any point $y$ in $F_{V}$ there is a curve $u:[0,1] \rightarrow F_{V}$ such that $u(0)=y_{*}$ and $u(1)=y$. Let $\tilde{u}$ be the lift of $\nu u$ starting from $\tilde{y}_{*}$ for the covering $\tilde{\nu}$. We define $i(y) \in \tilde{F}$ by $i(y)=\tilde{u}(1) . \quad i(y)$ is well defined, i.e. for another curve $v$ in $F_{V}$ from $y_{*}$ to $y, \tilde{v}(1)=\tilde{u}(1)$. In fact, since $\left[\nu\left(u v^{-1}\right)\right] \in G\left(F_{V}\right)$ and $G\left(F_{V}\right)=\tilde{\nu}_{*}\left(\pi_{1}\left(\tilde{F}, \tilde{y}_{*}\right)\right)$, the lift of $\nu\left(u v^{-1}\right)$ starting from $\tilde{y}_{*} \in \tilde{F}$ is a closed curve. Hence, $\tilde{u}^{-1} \tilde{v}$ is a closed curve with the base point $\tilde{u}(1)$. This implies $\tilde{v}(1)=\tilde{u}(1)$. By the definition of $i, \tilde{\nu} \circ i=\nu$ is obvious.

If $\nu(y) \neq \nu\left(y^{\prime}\right)$, clearly $i(y) \neq i\left(y^{\prime}\right)$. Next, we show that $i(y) \neq i\left(y^{\prime}\right)$ 
when $\nu(y)=\nu\left(y^{\prime}\right)$ and $y \neq y^{\prime}$. Let $u^{\prime}$ and $v^{\prime}$ be the curves in $F_{V}$ from $y_{*}$ to $y$ and $y^{\prime}$ respectively. Put $\nu u^{\prime}=u$ and $\nu v^{\prime}=v$. We can assume that $y<y^{\prime}$ in $\nu^{-1}(y)$. Since $h_{v-1}\left(y^{\prime}\right)=y_{*}, h_{v-1}(y)$ is defined and $h_{v-1}(y)$ $<y_{*}$ in $\nu^{-1}\left(x_{*}\right)$. Since $h_{u v^{-1}}\left(y_{*}\right)=h_{v^{-1}}(y)<y_{*}, \quad\left[u v^{-1}\right] \notin G\left(F_{V}\right)$. So that, the lift of $u v^{-1}$ starting from $\widetilde{y}_{*}$ in $\tilde{F}$ is never a closed curve. Hence, $i(y)=\tilde{u}(1) \neq \tilde{v}(1)=i\left(y^{\prime}\right)$. Therefore, $i$ is an injection.

It is obvious that $i$ maps any plaque of $F_{V} C^{r}$-diffeomorphically into $\tilde{F}$.

To show $\tilde{\nu}$ is a regular covering we are sufficient to show that $G\left(F_{V}\right)$ is a normal subgroup of $\pi_{1}\left(\boldsymbol{F}_{0}, x_{*}\right)$. Let $u$ and $v$ be closed curves in $F_{0}$ with the base point $x_{*}$. Assume $[u] \in G\left(F_{V}\right)$. Since $F_{V}$ is asymptotic to $F_{0}$ there is $y$ in $\nu^{-1}\left(x_{*}\right) \cap F_{\nu}$ such that $h_{v u v-1}(y)$ is defined. Since $[u]$ $\in G\left(F_{V}\right), h_{u} h_{v}(y)=h_{v}(y)$. So that, $h_{v v^{-1}}(y)=h_{v-1} h_{u} h_{v}(y)=y$. Hence, $\left[v u v^{-1}\right] \in G\left(F_{V}\right)$. This implies that $G\left(F_{V}\right)$ is a normal subgroup. Therefore, (i) is proved.

To prove (ii) it is sufficient, if $G\left(F_{V}\right)=G\left(F_{V^{\prime}}\right)$ is shown. But, this is obvious since $F_{V}$ is asymptotic to $F_{0}$.

Proof of Theorem 2. By Theorem 1 we obtain $N_{1}, \cdots, N_{\ell} \subset F_{0}, V$, and the functions $f_{1}, \cdots, f_{\ell}$. Let $x_{*} \in F_{0}-N_{1} \cup \cdots \cup N_{\ell}$. For an asymptotic leaf $F$ of $\mathscr{F} \mid V$ to $F_{0}$, let $F_{V}$ be an asymptotic leaf of $\mathscr{F} \mid V$ to $F_{0}$ such that $F_{V} \subset F$.

First, we show that, if $u, v$ are closed paths in $F_{0}$ with base point $x_{*}$ in a same homology class of $H_{1}\left(F_{0} ; Z\right), h_{u}(y)=h_{v}(y)$ for any $y \in \nu^{-1}\left(x_{*}\right)$ $\cap V$ such that $h_{u}(y), h_{v}(y)$ are defined. Let $\tilde{u}, \tilde{v}$ be the leaf curves of $\mathscr{F} \mid V$ which are lifts of $u, v$ starting from $y$. We may assume that $\tilde{u}, \tilde{v}$ intersect $\nu^{-1}\left(N_{1} \cup \cdots \cup N_{\ell}\right)$ transversely. So, since $F_{0}-N_{1} \cup \cdots \cup N_{\ell}$ is connected, $\tilde{u}$ and $\tilde{v}$ are homotopic to $\tilde{u}_{1} \cdots \tilde{u}_{\alpha}$ and $\tilde{v}_{1} \cdots \tilde{v}_{\beta}$ by homotopies such that the homotopies preserve the end points of the paths and that each homotopy level is a leaf curve of $\mathscr{F} \mid V$, where $\tilde{u}_{1} \cdots \tilde{u}_{\alpha}$ and $\tilde{v}_{1} \cdots \tilde{v}_{\beta}$ are the paths which are the compositions of the paths $\tilde{u}_{a}, \tilde{v}_{b}$ with end points in $\nu^{-1}\left(x_{*}\right)$ such that putting $\nu \tilde{u}_{a}=u_{a}$ and $\nu \tilde{v}_{b}=v_{b}, u_{a}$ and $v_{b}$ are closed paths in $F_{0}$ each of which intersect $N_{1} \cup \cdots \cup N_{\ell}$ at one point. Here, the composition of paths is defined by

$$
u v(x)= \begin{cases}u(2 t) & \text { for } 0 \leqq t \leqq \frac{1}{2} \\ v(2 t-1) & \text { for } \frac{1}{2} \leqq t \leqq 1\end{cases}
$$

Define $N_{i_{a}}$ and $N_{j_{b}}$ by $u_{a} \cap\left(N_{1} \cup \cdots \cup N_{\ell}\right)=u_{a} \cap N_{i_{a}}$ and $v_{b} \cap\left(N_{1}\right.$ 
$\left.\cup \cdots \cup N_{\ell}\right)=v_{b} \cap N_{j_{b}}$. Let the intersection numbers be $\left[u_{a}\right] \cdot\left[N_{i_{a}}\right]=\sigma_{a}$ and $\left[v_{b}\right] \cdot\left[n_{j_{b}}\right]=\tau_{b}$, where $\sigma_{a}, \tau_{b}= \pm 1$. Here, $N_{1}, \cdots, N_{\ell}$ are imposed the orientations such that if a closed path $u$ intersects $N_{1} \cup \cdots \cup N_{\ell}$ at only one point in $N_{i}$ with the intersection number $[u] \cdot\left[N_{i}\right]=\sigma$, as in the proof of Theorem 1, then the holonomy map $h_{u}$ of $u$ is coincide with $f_{i}^{\sigma}$. Thus, we have

$$
\begin{aligned}
h_{u}(y) & =h_{u_{1} \cdots h_{\alpha}}(y)=h_{u_{\alpha}} \cdots h_{u_{1}}(y) \\
& =f_{i_{\alpha}}^{\sigma_{\alpha}} \cdots f_{i_{1}}^{\sigma_{1}}(y) .
\end{aligned}
$$

Similarly,

$$
h_{v}(y)=f_{j_{\beta}}^{\tau_{\beta}} \cdots f_{j_{1}}^{\tau_{1}}(y) .
$$

Since $u$ and $v$ are in the same homology class, Lemma 5 implies $h_{u}(y)$ $=h_{v}(y)$.

If we can show that $V$ and $F_{V}$ satisfy the condition $(*)$ in Lemma 6 , the proof of Theorem 2 is completed by Lemma $6 .(*)$ is shown as follows. Let $y, z$ be two points in $\nu^{-1}\left(x_{*}\right) \cap F_{V}$ such that $h_{u}(y)$ and $h_{u}(z)$ are defined, where $u$ is a closed path in $F_{0}$ with end points $x_{*}$. We can assume $y \geqq h_{u}(y)$; if $y<h_{u}(y)$, consider the curve $u^{-1}$ with the inverse direction of $u$. Here, $<$ is considered in the coordinates $\nu^{-1}\left(x_{*}\right) \cap V$ $=x_{*} \times[0, \varepsilon]$. Let $y>z$. Since $h_{u}$ is a homomorphism, $h_{u}(y)>h_{u}(z)$. There is a path $\tilde{w}$ in $F_{V}$ from $y$ to $z$. Put $w=\nu \tilde{w}$. Since $h_{u}(y) \leqq y$ and $h_{w} h_{u}(y)$ is defined. $h_{u}(y) \leqq y$ implies $z=h_{w}(y) \geqq h_{w} h_{u}(y)$. Notice that $y=h_{u}(y)$ if and only if $z=h_{w} h_{u}(y)$. We have $h_{w-1 u w}(z)=h_{w} h_{u} h_{w-1}(z)$ $=h_{w} h_{u}(y)$. Since $w^{-1} u w$ and $u$ are in the same homology class, $h_{w-1 u v}(z)$ $=h_{u}(z)$ by the fact that we proved above. Thus, $h_{u}(z)=h_{w} h_{u}(y)$. Since $z=h_{w}(y)$, we have $y=h_{u}(y)$ if and only if $z=h_{u}(z)$. This proves Theorem 2.

\section{§. Proof of Theorem 3}

Let $\nu_{+}: U_{+}^{\prime} \rightarrow F_{0}$ be a collar. Since $\left\{\log h_{\alpha_{1}}^{\prime}, \cdots, \log h_{\alpha_{m}}^{\prime}\right\}$ is rationally independent, there is a closed curve $u$ in $F_{0}$ such that $h_{u}^{\prime} \neq 1$. We can assume that $0<h_{u}^{\prime}<1$. Let $x$ be the base point of $u$. There is an interval $[t, z)$ in $\nu_{+}^{-1}(x)$ and a positive number $r<1$ such that for any $y$ in $[x, z) h_{u}(y)$ is defined and that $h_{u}^{\prime}(y)<r$. Hence, $\lim _{i \rightarrow \infty}\left(h_{u}\right)^{i}(y)=x$ for any $y$ in $[x, z)$. Therefore, by taking a sufficiently small collar $U_{+}$, any leaf meeting $U_{+}$is asymptotic to $F_{0}$. We can $U_{-}$similarly. 
By the assumption of $\pi_{1}\left(F_{0}\right)$, the one sided holonomy group $\Phi_{\sigma}\left(F_{0}\right)$ is abelian for $\sigma=+$ or - . Let $V$ be any neighborhood of $F_{0}$ in $U_{\sigma}$. Then, for any leaf $F$ meeting $U_{\sigma}$ a regular covering $\tilde{\nu}: \tilde{F} \rightarrow F_{0}$ is associated with $F_{V}$, by Theorem 2 .

Since holonomy has no torsion element, $G(F)=\nu_{*} \pi_{1}\left(F_{V}\right)=\tilde{\nu}_{*} \pi_{1}(\tilde{F})$ $\supset G . \quad \nu_{*}$ and $\tilde{\nu}_{*}$ are injections. Suppose that there is a leaf $F$ such that, for the associated covering $\tilde{\nu}: \tilde{F} \rightarrow F_{0}$ with $F_{V}, G(F) \neq G$. Then, there is a closed curve $\tilde{u}$ in $\tilde{F}$ with base point in $\tilde{\nu}^{-1}(x)$ such that the homotopy class $\alpha=[\tilde{\nu} \tilde{u}]$ is not contained in $G$. By the definition of $\tilde{F}$, there is a closed curve $u$ in $F_{V}$ starting from a point $y$ in $\nu_{\sigma}^{-1}(x)$ such that $\left[\nu_{o} u\right]=\alpha$. Then, for any $y^{\prime}$ in the interval $[x, y]$ in $\nu_{o}^{-1}(x)$, the holonomy map $h_{\alpha}\left(y^{\prime}\right)$ is defined. As above, there is a sequence of points $y_{0}=y, y_{1}, y_{2}, \cdots$ in $[x, y] \cap F_{V}$ such that $\lim _{i \rightarrow \infty} y_{i}=x$. By condition (*) of Lemma 6, $h_{\alpha}\left(y_{i}\right)=y_{i}$ for each $y_{i}$. Since $\pi_{1}\left(F_{0}, x\right)=Z_{(1)}+\cdots+Z_{(m)}+G$, and $\nu_{*} \pi_{1}\left(F_{V}\right) \supset G$, we can put

$$
\alpha=a_{1} \alpha_{1}+a_{2} \alpha_{2}+\cdots+a_{m} \alpha_{m}
$$

for the integers $a_{1}, \cdots, a_{m}$ with $\left(a_{1}, \cdots, a_{m}\right) \neq(0, \cdots, 0)$. Let $u_{1}, \cdots, u_{m}$ be the closed curves with base point $x$ realizing the homotopy classes $\alpha_{1}, \cdots, \alpha_{m}$ respectively. Then, the multiple $v=u_{1}^{a_{1}} \cdots u_{m}^{a_{m}}$ realizes $\alpha$, so that, $[v]=\left[\nu_{\sigma} u\right]$. Let $v_{t}$ be a homotopy from $u$ to $v, t \in[0,1]$. Since $h_{v_{t}}\left(y^{\prime}\right)$ is defined for arbitrary $y^{\prime} \in[x, y]$ which is sufficiently close to $x$, we have $h_{u}\left(y^{\prime}\right)=h_{v}\left(y^{\prime}\right)$. Hence, for such $y^{\prime}$

$$
h_{\alpha}\left(y^{\prime}\right)=\left(h_{\alpha_{m}}\right)^{a_{m}} \cdots\left(h_{\alpha_{1}}\right)^{a_{1}}\left(y^{\prime}\right) .
$$

Since $\lim _{i \rightarrow \infty} y_{i}=x$ and $h_{\alpha}\left(y_{i}\right)=y_{i}$, we have $h_{\alpha}^{\prime}=1$. Hence,

$$
\left(h_{\alpha_{m}}^{\prime}\right)^{a_{m}} \cdots\left(h_{\alpha_{1}}^{\prime}\right)^{a_{1}}=1 .
$$

Therefore,

$$
a_{1} \log h_{\alpha_{1}}^{\prime}+\cdots+a_{m} \log h_{\alpha_{m}}^{\prime}=0
$$

with $\left(a_{1}, \cdots, a_{m}\right) \neq(0, \cdots, 0)$. But, this contradicts to the assumption of the theorem. This proves Theorem 3 .

\section{§6. Proof of Theorem 4}

The proof of (i) and (ii) of Theorem 4 is contained in the proof of Theorem 3. 
Next we prove (iii). Since $f$ is a local diffeomorphism of class $C^{2}$ with $f(0)=0$ and $f^{\prime}(0)>1$, by a theorem of Sterenberg [5], there is a $C^{1}$-diffeomorphism $g$ from a neighborhood of 0 of $\boldsymbol{R}$ into $\boldsymbol{R}$ such that $f^{\prime}(0) \cdot t=g f g^{-1}(t)$ for any $t$ in the image of $g$. Hence, by a $C^{1}$-alternation of the coordinate of $\nu^{-1}(x) \cap U$, we may assume that $f(t)=d t$, where $d=f^{\prime}(0)<1$. Hereafter we use the new coordinate of $\nu^{-1}(x) \cap U$ translated by $g$. Let $f_{1}, \cdots, f_{\ell}$ be local diffeomorphisms of $\boldsymbol{R}$ generating $\Phi\left(F_{0}\right)$. Since $\Phi\left(F_{0}\right)$ is abelian, we may assume $f_{i} f=f f_{i}$ for $i=1$, $\cdots, m$ by choosing $U$ sufficiently small. Hence, $f_{i}^{\prime}(f(t)) \cdot f^{\prime}(t)=f^{\prime}\left(f_{i}(t)\right)$ $\cdot f_{i}^{\prime}(t)$, and so $f_{i}^{\prime}(f(t))=f_{i}^{\prime}(t)$, for $f^{\prime}(t)=d$. Then, $f_{i}^{\prime}(t)=f_{i}^{\prime}(0)$, since $\lim _{n \rightarrow \infty} f^{n}(t)=0$ and $f_{i}$ is of class $C^{1}$. Therefore, $f_{i}(t)=d_{i} \cdot t$, where $d_{i}$ $=f_{i}^{\prime}(0)$. To show (iii), it is sufficient if $G(F)=G\left(F^{\prime}\right)$ is shown for any asymptotic leaves $F$ and $F^{\prime}$ to $F_{0}$. Let $\alpha$ be a closed curve realizing an element of $G(F)$ and let $h_{\alpha}$ be the holonomy map defined by $\alpha \in \pi_{1}\left(F_{0}, x\right)$. Then, $h_{\alpha}$ can be written as $h_{\alpha}=f_{1}^{k_{1}} \cdots f_{\ell}^{k_{\ell}}$. By the definition of $G(F)$, there is a closed curve $\beta$ in $F \cap U$ with the end point $t$ in $\nu^{-1}(x)$ such that $\alpha=\nu \circ \beta$. Hence, $t=h_{\alpha}(t)=f_{1}^{k_{1}} \cdots f_{\ell}^{k_{\ell}}(t)=d_{1}^{k_{1}} \cdots d_{\ell}^{k_{\ell}} \circ t$. Thus, $h_{\alpha}=i d$. since $d_{1}^{k_{1}} \cdots d_{\ell}^{k_{\ell}}=1$. Therefore, a lift of $\alpha$ to $F^{\prime}$ is a closed curve, and so the holonomy class of $\alpha$ is contained in $G\left(F^{\prime}\right)$. This implies $G(F)$ $=G\left(F^{\prime}\right)$. This completes the proof of Theorem 3 .

Remark 1. For $\tilde{f} \in \Phi\left(F_{0}\right)$ let $\tilde{f}^{\prime} \in \boldsymbol{R}$ be the derivative of $\tilde{f}$ at 0 . Denoting $D \Phi\left(F_{0}\right)=\left\{\tilde{f}^{\prime} \mid \tilde{f} \in \Phi\left(F_{0}\right)\right\}, D \Phi\left(F_{0}\right)$ is a multiplicative subgroup of $R-\{0\}$. Let $D: \Phi\left(F_{0}\right) \rightarrow D \Phi\left(F_{0}\right)$ be the homomorphism defined by the derivation. Then, for any asymptotic leaf $F$ to $F_{0}$, we see that $G(F)$ $\subset$ ker $D h$, where $h$ is the homomorphism $\pi_{1}\left(F_{0}, x_{*}\right) \rightarrow \Phi\left(F_{0}\right)$ defined in $\S 3$.

Remark 2. If $\mathscr{F}$ is of class $C^{2}$, then, by the method used in the proof of Theorem 4, we see that the sequence

$$
1 \longrightarrow G(F) \stackrel{\subset}{\longrightarrow} \pi_{1}\left(F_{0}\right) \stackrel{h}{\longrightarrow} \Phi\left(F_{0}\right) \longrightarrow 1
$$

is exact for any asymptotic leaf $F$ to $F_{0}$.

\section{§ 7. Proof of Theorem 5}

Assuming that $\pi_{1}(F)=Z_{(1)}+\cdots+Z_{(m)}+G$ for a finite group $G$, let $N_{1}, \cdots, N_{m}$ be the manifolds of $F$ obtained by Proposition 1 for the isomorphism $H: H_{1}(F: Z) \rightarrow Z_{(1)}+\cdots+Z_{(m)}$. Here, we may assume 
that $\operatorname{dim} F \geqq 2$, because if $\operatorname{dim} F=2, F$ is a torus. By observing the proof of Theorem 1, the same conclusion of Theorem 1 is satisfied for these $N_{1}, \cdots, N_{m}$. Then, if $\mathscr{F}$ is a foliation of class $C^{r}$, there are injective $C^{r}$-diffeomorphisms $f_{i}^{+}:[0, \varepsilon] \rightarrow[0, \varepsilon]$ for $i=1, \cdots, m$ with the properties (a) and (b) of Theorem 1. By the proof of Theorem 1, $f_{i}$ can be identified with an one sided holonomy map $h_{\alpha_{i}}^{+}$of a generator $\alpha_{i}$ of $Z_{(i)}$.

We divide the stage into Case 1 and Case 2. (i) of Theorem 5 is divided into the both cases and (ii) is contained in Case 1.

Case 1: The case that $\mathscr{F}$ is of class $C^{r}, r \geqq 2$, and that there is $i$ such that $\left(f_{i}^{+}\right)^{\prime}(0) \neq 1$. Let $f_{j}$ be a (both sided) holonomy map of $\alpha_{j}$. Then $f_{j}^{\prime}(0)=\left(f_{j}^{+}\right)^{\prime}(0)$. By Sternberg's theorem, $f_{1}, \cdots, f_{m}$ are $C^{r-1}$-conjugate to linear functions by a same conjugation map $g$ in a small neighborhood of 0 . (See the proof of Theorem 4.) Then, $g f_{j} g^{-1}(t)$ $=f_{j}^{\prime}(0) \cdot t$ if $|t|$ is sufficiently small. Let $U_{-}$be a collar of $F$ such that $U_{-}$is in the another side of $U_{+}$. Using Theorem 1 we get $f_{i}^{-}:\left[-\varepsilon^{\prime}, 0\right]$ $\rightarrow\left[-\varepsilon^{\prime}, 0\right]$ for $i=1, \cdots, m . f_{i}^{-}$is the other sided holonomy map of a generator $\alpha_{i}^{\prime}$ of $Z_{(i)} \cdot\left|f_{i}(t)\right| \leqq|t|$ for sufficiently small $|t|$ if and only if $\left|f_{i}^{\prime}(0)\right| \leqq 1$ since $\bar{f}_{i}=g f_{i} g^{-1}$ is linear and $\bar{f}_{i}(t)=f_{i}^{\prime}(0) \cdot t, i=1, \cdots, m$. Hence, by taking $\varepsilon^{\prime}$ small, $\alpha_{i}^{\prime}=\alpha_{i}$, i.e. $f_{i}^{+}$and $f_{i}^{-}$are the one sided holonomies of the same generator $\alpha_{i}$ of $Z_{(i)}$. Therefore, there are injective linear maps $\bar{f}_{i}:[-\varepsilon, \varepsilon] \rightarrow[-\varepsilon, \varepsilon], i=1, \cdots, m$ with the following properties: Let $N_{i}^{\prime}, N_{i}^{\prime \prime}$, and $F_{*}$ be the manifolds defined in Theorem 1 . Denote by $X_{\bar{f}}$ the quotient manifold obtained from $F_{*} \times[-\varepsilon, \varepsilon]$ by identifying $(x, t) \in N_{i}^{\prime} \times[-\varepsilon, \varepsilon]$ and $\left(x, \bar{f}_{i}(t)\right) \in N_{i}^{\prime \prime} \times[-\varepsilon, \varepsilon]$ for all $i=1, \cdots, m$ and $t \in[-\varepsilon, \varepsilon]$. The product foliation of $F_{*} \times[-\varepsilon, \varepsilon]$ induces a foliation $\mathscr{F}_{\bar{f}}$ on $X_{\bar{f}}$. Then, there is a neighborhood $V$ of $F$ such that there is a leaf preserving $C^{r-1}$-diffeomorphism $\varphi$ from $V$ onto $X_{\tilde{f}}$ which maps $F$ onto $F_{*} \times 0 / \sim$.

By Theorem 4, for any leaf $F^{\prime}$ meeting $V$ an unique regular covering $\tilde{F}$ is associated with $F_{V}^{\prime}$. Since $\bar{f}_{i}$ is linear, by Theorem $3, \nu_{*} \pi_{1}\left(F_{V}^{\prime}\right)$ $=\tilde{\nu}_{*} \pi_{1}(\tilde{F}) \cong \pi_{1}(\tilde{F}) \cong G$ if and only if $\log \bar{f}_{1}^{\prime}, \cdots, \log \bar{f}_{m}^{\prime}$ are rationally independent. By an arbitrarily small perturbations of $\bar{f}_{1}, \cdots, \bar{f}_{m}$, we can take linear maps $\bar{g}_{1}, \cdots, \bar{g}_{m}:[-\varepsilon, \varepsilon] \rightarrow[-\varepsilon, \varepsilon]$ such that $\log \bar{g}_{1}^{\prime}, \cdots, \log \bar{g}_{m}^{\prime}$ are rationally independent or dependent when $\log \bar{f}_{1}^{\prime}, \cdots, \log \bar{f}_{m}^{\prime}$ are rationally dependent or independent, respectively.

Let $U$ be an open neighborhood of $F$ contained in $V$. Let $N$ be a 
neighborhood of $\mathscr{F} \mid U$ in $\mathrm{Fol}_{F}^{r-1}(U), \quad \varphi(U)$ is a neighborhood of $F_{*} \times 0 / \sim$ in $X_{\bar{f}}$. Since $\bar{g}_{1}, \cdots, \bar{g}_{m}$ are close to $\bar{f}_{1}, \cdots, \bar{f}_{m}$ we may assume that $\varphi(U) \subset X_{\bar{g}} \subset X_{\bar{f}}$ and that $\mathscr{F}_{\bar{g}} \mid \varphi(U)$ is close to $\mathscr{F}_{\bar{f}}\left|\varphi(U) . \mathscr{F}_{g}\right| \varphi(U)$ induce a foliation $\mathscr{F}^{\prime}$ of $U$. By taking $\bar{g}_{1}, \cdots, \bar{g}_{m}$ sufficiently close to $\bar{f}_{1}, \cdots, \bar{f}_{m}$ we can $\mathscr{F}^{\prime} \in N$.

Case 2. The case that $\mathscr{F}$ is of class $C^{1}$ and that $\left(f_{i}^{+}\right)^{\prime}(0)=1$ for all $i=1, \cdots, m . \quad f_{i}^{+}$is the one sided holonomy map of $\alpha_{i}$ defined on $[0, \varepsilon]$. First, assume that there is no neighborhood $U$ of $F$ such that $F \mid U$ is a product foliation. For small $\delta>0$ we define a $C^{1}$-diffeomorphism $g_{i}^{+}:[0, \varepsilon+\delta] \rightarrow \boldsymbol{R}_{+}$by

$$
g_{i}^{+}(t)= \begin{cases}t & \text { for } 0 \leqq t<\delta \\ f_{i}^{+}(t-\delta)+\delta & \text { for } t>\delta .\end{cases}
$$

Since $\left(f_{i}^{+}\right)^{\prime}(0)=1, g_{i}^{+}$is of class $C^{1}$. It is easy to see that $g_{1}^{+}, \cdots, g_{m}^{+}$ are mutually commutative since $f_{1}^{+}, \cdots, f_{m}^{+}$are so. $g_{i}^{+} \mid[0, \varepsilon]$ is a $C^{1}$ perturbation of $f_{i}^{+}$. Let $\mathscr{F}_{f}$ and $X_{f}$ be the ones defined in Theorem 1 from $f_{i}^{+}$and $F_{*} \times[0, \varepsilon]$. Define $\mathscr{F}_{g}$ and $X_{g}$ similarly from $g_{i}^{+}$and $F_{*} \times[0, \varepsilon+\delta]$. We can consider that $X_{f} \subset X_{g}$ and that $\mathscr{F}_{g} \mid X_{f}$ is $C^{1}$ close to $\mathscr{F}_{f}$ is $\delta$ is small enough. There is a neighborhood $V_{+}$of $F$ in $U_{+}$and a $C^{1}$-diffeomorphism $\varphi: V_{+} \rightarrow X_{f}$ mapping $\mathscr{F} \mid V_{+}$to $\mathscr{F}_{f}$. Let $\mathscr{F}_{+}^{\prime}$ be the foliation induced by $\varphi^{-1}$ from $\mathscr{F}_{g} \mid X_{f} . \mathscr{F}_{+}^{\prime}$ is $C^{1}$-close to $\mathscr{F} \mid V_{+}$ if $\delta$ is small enough. We get $\mathscr{F}_{-}^{\prime}$ on $V_{-}$similarly. On small neighborhoods of $F, \mathscr{F}_{+}^{\prime}$ and $\mathscr{F}_{-}^{\prime}$ are product foliations. Let $U=V_{+} \cup V_{-}$. Then, we get $\mathscr{F}^{\prime}$ on $U$ by $\mathscr{F}^{\prime} \mid V_{\sigma}=\mathscr{F}_{\sigma}^{\prime}, \sigma= \pm$. We can take $\mathscr{F}^{\prime}$ in any neighborhood $N$ of $\mathscr{F} \mid U$ in $\mathrm{Fol}_{F}^{1}(U)$. By the assumption $\mathscr{F}^{\prime}$ is not locally equivalent to $\mathscr{F} \mid U$.

Next, we assume that there is a neighborhood $V$ of $F$ such that $\mathscr{F} \mid V$ is a product foliation. Then, $V$ is leaf preservingly diffeomorphic to $F \times[-\varepsilon, \varepsilon]$. Consider that $V=F \times[-\varepsilon, \varepsilon]$ and $F=F \times 0$. Let $U$ $=F \times(-\varepsilon / 2, \varepsilon / 2)$. Let $\alpha_{i}$ be a generator of $Z_{(i)}$. Then, the holonomy map $f_{i}:[-\varepsilon, \varepsilon] \rightarrow[-\varepsilon, \varepsilon]$ of $\alpha_{i}$ is the identity map. Let $g_{i}$ be the perturbation of $f_{i}$ such that $g_{i}=f_{i}$ for $i>1$ and that $\left|g_{1}(t)\right|\left\langle|t|\right.$ and $\left|g_{1}( \pm \varepsilon)\right|>\varepsilon / 2$. Let $\mathscr{F}_{g}$ and $X_{g}$ be as above defined from $g_{i}$ and $F_{*} \times[-\varepsilon, \varepsilon]$. Then, we can consider that $U \subset X_{g} \subset V$ and that $\mathscr{F}_{g} \mid U$ is close to $\mathscr{F} \mid U$ if $g_{1}$ is close enough to $f_{1}$. Any leaf of $\mathscr{F}_{g}$ is asymptotic to $F$, but any leaf of $\mathscr{F} \mid V$ is not asymptotic. Hence, $\mathscr{F}_{g} \mid U$ is not locally equivalent to $\mathscr{F} \mid V$. This completes the proof of Theorem 5 . 


\section{REFERENCES}

[1] M. Hirsch, Stability of compact leaves of foliations, Dynamical Systems, ed. M. Peixoto, Academic Press, N.Y., 1973, 135-153.

[2] H. Levine and M. Shub, Stability of foliations, Trans. A.M.S., 184 (1973), 419437.

[ 3 ] H. Nakatsuka, On representations of homology classes, Proc. Japan Acad., 48 (1972), 360-364.

[4] T. Nishimori, Compact leaves with abelian holonomy, Tōhoku Math. J. The Second Series, 27 (1975), 259-272.

[ 5 ] S. Sterenberg, Local $C^{n}$ transformation of the real line, Duke Math. J., 24 (1957), $97-102$.

Department of Mathematics

College of General Education

Nagoya University 